\title{
ON DISCONJUGACY AND $k$-POINT DISCONJUGACY FOR LINEAR ORDINARY DIFFERENTIAL OPERATORS
}

\author{
JAMES S. MULDOWNEY ${ }^{1}$
}

\begin{abstract}
It is shown that a linear ordinary differential operator of order $n$ which is $k$-point disconjugate on an open interval for some $k, 2 \leq k \leq n$, is also disconjugate on that interval.
\end{abstract}

1. Introduction. Consider the linear differential operator $L$ of order $n$ defined by

$$
L u=u^{(n)}+a_{1}(t) u^{(n-1)}+\cdots+a_{n}(t) u
$$

where $a_{i}, i=1, \ldots, n$, are continuous real valued functions on an open interval $I$. The operator $L$ is said to be disconjugate on an interval $J \subset I$ if the only solution of the equation $L u=0$ having $n$ zeros or more in $J$, counting multiplicities, is the zero solution. The operator is said to be $k$-point disconjugate on $J$ if the zero solution is the only solution of $L u=0$ having zeros at $k$ distinct points in $J$, the sum of whose multiplicities is not less than $n$.

It is clear that, for any interval $J$, disconjugacy implies $k$-point disconjugacy. Conversely, for open intervals, it is a special case of a result of Hartman [1] that $n$-point disconjugacy implies disconjugacy and Sherman [4] showed that 2-point disconjugacy implies disconjugacy for any interval. The question of whether $k$ point disconjugacy $(2<k<n)$ implies disconjugacy on open intervals has been addressed by Henderson [2] who showed that this implication holds subject to strong additional restrictions on $k$. This paper removes these restrictions.

2. Result. We prove the following theorem.

THEOREM 2.1. Suppose $2 \leq k \leq n$ and $L$ is $k$-point disconjugate on the open interval $I$. Then $L$ is disconjugate on $I$.

The proof will be furnished by introducing some notation and establishing the validity of a number of assertions. In view of the result of Sherman [4], it is sufficient to prove the theorem for $2<k \leq n$.

Let $u_{1}, \ldots, u_{n}$ be a fundamental solution set for the equation $L u=0$ such that the wronskian determinant $W\left(u_{1}, \ldots, u_{n}\right)=\operatorname{det}\left[u_{i}^{(j-1)}\right]$ is positive on $I$. If $\left\{\tau_{1}, \ldots, \tau_{k}\right\} \subset I$ and $m_{1}, \ldots, m_{k}$ are nonnegative integers such that $m_{1}+\cdots+m_{k}=$ $n$, let $\mathcal{W}\left(\tau_{1}, m_{1} ; \tau_{2}, m_{2} ; \ldots ; \tau_{k}, m_{k}\right)$ denote the $n \times n$ matrix of which the $i$ th column, $i=1, \ldots, n$, is

$$
\operatorname{col}\left[u_{i}\left(\tau_{1}\right), \ldots, u_{i}^{\left(m_{1}-1\right)}\left(\tau_{1}\right), u_{i}\left(\tau_{2}\right), \ldots, u_{i}^{\left(m_{2}-1\right)}\left(\tau_{2}\right), \ldots, u_{i}\left(\tau_{k}\right), \ldots, u_{i}^{\left(m_{k}-1\right)}\left(\tau_{k}\right)\right] .
$$

Received by the editors July 20, 1983.

1980 Mathematics Subject Classification. Primary 34C10.

Key words and phrases. Linear differential equation, disconjugacy.

${ }^{1}$ Research supported by NSERC grant 7197 . 
If $m_{j}=0$ there are no terms corresponding to the point $\tau_{j}$. Note that $\operatorname{det} \mathcal{W}(\tau, n)=$ $W\left(u_{1}, \ldots, u_{n}\right)(\tau)$.

Suppose that $L$ is not disconjugate on $I$. Then there exist points $\alpha, \gamma \in I$ such that $\gamma$ is the first right conjugate point of $\alpha$, which means $L$ is not disconjugate on $[\alpha, \gamma]$, but is disconjugate on every proper subinterval of $[\alpha, \gamma]$. Let $\beta \in(\alpha, \gamma)$.

ASSERTION 2.2. There exist integers $p, q, r$ with $p>0, q \geq 0, r>0, p+q+r=n$ such that

$$
\operatorname{rank} \mathcal{W}(\alpha, p ; \beta, q ; \gamma, r)<n
$$

Assertion 2.2 is equivalent to the existence of a nonzero solution of $L u=0$ having $p$ zeros at $\alpha, q$ zeros at $\beta$ and $r$ zeros at $\gamma$. The existence of such a solution was established by Sherman [4] with $q=0$.

ASSERTION 2.3 First choose $q$ to be the largest integer such that (1) holds. Next let $r$ be maximal such that (1) holds for this choice of $q$ and let $p=n-q-r$.

(a) At least one of the cofactors of the last row, $\operatorname{row}\left[u_{1}^{(r-1)}(\gamma), \ldots, u_{n}^{(r-1)}(\gamma)\right]$, in $\mathcal{W}(\alpha, p ; \beta, q ; \gamma, r)$ is nonzero.

(b) There is a solution $u$ of $L u=0$ which has $p$ zeros at $\alpha, q$ zeros at $\beta$ and $r$ zeros at $\gamma, u^{(r)}(\gamma) \neq 0$, and which is unique to within a constant multiple.

To prove Assertion 2.3(a), observe that, if these cofactors were all zero, this would imply

$$
\operatorname{rank} \mathcal{W}(\alpha, p ; \beta, q+1 ; \gamma, r-1)<n,
$$

contradicting the maximal character of $q$ if $r-1>0$ and contradicting the disconjugacy of $L$ on $[\alpha, \beta]$ if $r-1=0$.

Assertion 2.3(b) follows from the fact that (1) implies the existence of a nontrivial solution $u$ of $L u=0$ which has $p$ zeros at $\alpha, q$ zeros at $\beta$ and $r$ zeros at $\gamma$. If $u^{(r)}(\gamma)=0$ for any such solution, then

$$
\operatorname{rank} \mathcal{W}(\alpha, p-1 ; \beta, q ; \gamma, r+1)<n .
$$

But, if $p-1>0,(2)$ contradicts the maximality of $r$; if $p-1=0, q>0,(2)$ contradicts the disconjugacy of $L$ on $[\beta, \gamma]$; if $p-1=0, q=0,(2)$ contradicts $W\left(u_{1}, \ldots, u_{n}\right)(\gamma)>0$. Thus $u^{(r)}(\gamma) \neq 0$. To see that $u$ is unique, note that the existence of two independent solutions with $p$ zeros at $\alpha, q$ zeros at $\beta$ and $r$ zeros at $\gamma$ would imply the existence of such a solution with $u^{(r)}(\gamma)=0$ which, as we have proved, leads to a contradiction.

ASSERTION 2.4. Let $p, q, r$ be chosen as in Assertion 2.3. Then the function

$$
F(t)=\operatorname{det} \mathcal{W}(\alpha, p ; \beta, q ; t, r)
$$

changes sign at $t=\gamma$.

We prove this assertion by considering the function

$$
u(t)=\operatorname{det} \mathcal{W}(\alpha, p ; \beta, q ; \gamma, r-1 ; t, 1),
$$

which is a solution of $L u=0$. It is a nontrivial solution, by Assertion 2.3(a), and clearly

$$
\begin{array}{ll}
u^{(i-1)}(\alpha)=0, & i=1, \ldots, p \\
u^{(i-1)}(\beta)=0, & i=1, \ldots, q \\
u^{(i-1)}(\gamma)=0, & i=1, \ldots, r-1
\end{array}
$$


also $u^{(r-1)}(\gamma)=\operatorname{det} \mathcal{W}(\alpha, p ; \beta, q ; \gamma, r)=0$, from (1). Thus $u$ is the unique solution of $L u=0$ whose existence is Assertion 2.3(b). Now

$$
F(\gamma)=\operatorname{det} \mathcal{W}(\alpha, p ; \beta, q ; \gamma, r)=0,
$$

from (1), and $F^{\prime}(\gamma)=u^{(r)}(\gamma) \neq 0$, from Assertion 2.3(b). Therefore $F$ changes sign at $\gamma$.

ASSERTION 2.5. Let $2<k \leq n$ and let $F$ be the function defined in Assertion 2.4. If $L$ is $k$-point disconjugate on $I$, then $F(t) \geq 0$ for all $t>\beta, t \in I$.

The $k$-point disconjugacy of $L$ on $I$ implies that

$$
\operatorname{det} \mathcal{W}\left(\tau_{1}, m_{1} ; \ldots ; \tau_{k}, m_{k}\right) \neq 0
$$

if $m_{i}>0, \tau_{i} \in I, i=1, \ldots, k, \tau_{1}<\tau_{2}<\cdots<\tau_{k}$. Continuity in $\left(\tau_{1}, \ldots, \tau_{k}\right)$ implies that, for any choice of $\left(m_{1}, \ldots, m_{k}\right)$, all of the determinants considered in (3) are of one sign. They are in fact positive since, by successive applications of the Mean Value Theorem, $\operatorname{det} \mathcal{W}\left(\tau_{1}, m_{1} ; \cdots ; \tau_{k}, m_{k}\right)$ is a positive multiple of a determinant of the form $\operatorname{det}\left[u_{i}^{(j-1)}\left(\alpha_{j}\right)\right], i, j=1, \ldots, n$, where $\tau_{1}=\alpha_{1} \leq \alpha_{2} \leq \cdots \leq \alpha_{n}<$ $\tau_{k}$. When $\tau_{1}, \ldots, \tau_{k}$ are all close to a point $\tau$, then so also are $\alpha_{1}, \ldots, \alpha_{n}$ and $\operatorname{det} W\left(\tau_{1}, m_{1} ; \cdots ; \tau_{k}, m_{k}\right)>0$ follows from continuity and $W\left(u_{1}, \ldots, u_{n}\right)(\tau)>0$. Next, choose $m_{1}, \ldots, m_{k}, h, j$ such that $m_{i}>0, i=1, \ldots, k, 1 \leq h \leq j<k$ and

$$
m_{1}+\cdots+m_{h}=p, \quad m_{h+1}+\cdots+m_{j}=q, \quad m_{j+1}+\cdots+m_{k}=r .
$$

The case $h=j$ corresponds to $q=0$ and, in this case, there are no terms $m_{h+1}, \ldots, m_{j}$ present. Again by Rolle's Theorem, if $\tau_{1}<\tau_{2}<\cdots<\tau_{k}$, the positive determinant $\operatorname{det} \mathcal{W}\left(\tau_{1}, m_{1} ; \cdots ; \tau_{k}, m_{k}\right)$ is a positive multiple of the determinant $\Delta\left(\alpha_{1}, \ldots, \alpha_{p}, \beta_{1}, \ldots, \beta_{q}, t_{1}, \ldots, t_{r}\right)$ of a $n \times n$ matrix of which the $i$ th column has the form

$$
\operatorname{col}\left[u_{i}\left(\alpha_{1}\right), \ldots, u_{i}^{(p-1)}\left(\alpha_{p}\right), u_{i}\left(\beta_{1}\right), \ldots, u_{u}^{(q-1)}\left(\beta_{q}\right), u_{i}\left(t_{1}\right), \ldots, u_{i}^{(r-1)}\left(t_{r}\right)\right],
$$

$i=1, \ldots, n$, for some numbers $\alpha_{1}, \ldots, \alpha_{p}, \beta_{1}, \ldots, \beta_{q}, t_{1}, \ldots, t_{r}$ such that

$$
\begin{aligned}
\tau_{1} & =\alpha_{1} \leq \alpha_{2} \leq \cdots \leq \alpha_{p} \leq \tau_{h}, \\
\tau_{h+1} & =\beta_{1} \leq \beta_{2} \leq \cdots \leq \beta_{q} \leq \tau_{j}, \\
\tau_{j+1} & =t_{1} \leq t_{2} \leq \cdots \leq t_{r} \leq t_{k},
\end{aligned}
$$

and therefore

$$
\Delta\left(\alpha_{1}, \ldots, \alpha_{p}, \beta_{1}, \ldots, \beta_{q}, t_{1}, \ldots, t_{r}\right)>0
$$

for these numbers. But, if

$$
\left(\alpha_{1}, \ldots, \alpha_{p}\right) \rightarrow(\alpha, \ldots, \alpha), \quad\left(\beta_{1}, \ldots, \beta_{q}\right) \rightarrow(\beta, \ldots, \beta), \quad\left(t_{1}, \ldots, t_{r}\right) \rightarrow(t, \ldots, t),
$$

then

$$
\Delta\left(\alpha_{1}, \ldots, \alpha_{p}, \beta_{1}, \ldots, \beta_{q}, t_{1}, \ldots, t_{r}\right) \rightarrow \operatorname{det} \mathcal{W}(\alpha, p ; \beta, q ; t, r)=F(t) .
$$

Thus, by considering $\tau_{1}, \ldots, \tau_{k}$ close to $\alpha, \tau_{h+1}, \ldots, \tau_{j}$ close to $\beta$ and $\tau_{j+1}, \ldots, \tau_{k}$ close to $t$, we see from (4) and (5) that $F(t) \geq 0$ if $t>\beta$, proving Assertion 2.5.

Considering Assertions 2.4 and 2.5, we see that the open interval $I$ cannot contain a point $\alpha$ and its first right conjugate point $\gamma$ while $L$ is $k$-point disconjugate on $I$. This proves Theorem 2.1. 
3. Remarks. An examination of the proof of Assertion 2.5 shows that Theorem 2.1 is still true if a more stringent definition of $k$-point disconjugacy is adopted. Specifically, if $2 \leq k \leq n$ and, for each set of points $t_{1}, \ldots, t_{k}$ in I with $t_{1}<t_{2}<$ $\cdots<t_{k}, u=0$ is the only solution of $L u=0$ which has a zero of multiplicity $m_{i}>0$ at $t_{i}, i=1, \ldots, k$, where $m_{1}+m_{k} \geq n-k+2$, then $L$ is disconjugate on $I$. In the case $k=2$, this is again implied by Sherman's result [4] and, when $2<k \leq n$, Assertion 2.5 may be proved with $m_{2}=m_{3}=\cdots=m_{k-1}=1$.

With minor modifications, the proof given here may be adapted to provide similar results in the case of disfocality and generalizations thereof; cf. [3].

ACKNOWLEDGEMENT. This paper was motivated by conversations with Uri Elias.

\section{REFERENCES}

1. P. Hartman, Unrestricted n-parameter families, Rend. Circ. Mat. Palermo (2) 7 (1958), 123-142.

2. J. Henderson, k-point disconjugacy and disconjugacy for linear differential equations, Univ. of Missouri (preprint 1982).

3. J. S. Muldowney, On invertibility of linear ondinary differential boundary value problems, SIAM J. Math. Anal. 12 (1981), 368-384.

4. T. L. Sherman, Properties of solutions of $n$th order linear differential equations, Pacific J. Math. 15 (1965), 1045-1060.

Department of Mathematics, University of Alberta, Edmonton, Alberta, CANADA, T6G 2G1 